\title{
Modeling GaAs/AlGaAs Devices: A Critical Review
}

\author{
Herbert S. Bennett
}

\begin{abstract}
Device models for GaAs devices and GaAs/AlGaAs heterostructures are much less advanced than those for silicon devices. This paper critically reviews recent advances in the modeling of $\mathrm{GaAs} / \mathrm{AlGaAs}$ devices. It is based on the examination of five selected device models that contain features common to the majority of device models for heterostructure bipolar and field effect transistors. Areas requiring improved measurement techniques on processed $G a A s$ and improved physical concepts for $G a A s / A l G a A s$ device models are identified.
\end{abstract}

\section{Introduction}

This review summarizes recent advances in modeling GaAs/AlGaAs heterostructure bipolar transistors (HBT) and GaAs and GaAs/AlGaAs field effect transistors (FET). It identifies those physical concepts that are not adequately included in present device models for HBTs, for conventional metal semiconductor FETs (MESFETs), and for heterostructure FETs (HFETs) such as two-dimensional electron gas FETs (TEGFETs), modulation doped FETs (MODFETs), high electron mobility FETs (HEMTs), and selectively doped FETs (SDFETs). This review also identifies areas requiring increased efforts for measurement techniques on processed GaAs. The term processed $\mathrm{GaAs}$ refers to material that is representative of the active regions of devices and not to bulk material. This review is based on the examination of five device models that have been selected from among several available GaAs device models. The five device models discussed contain features and assumptions found in the majority of device models reported in the archival literature.

Developing computationally efficient models that simulate the operation of solid-state devices is one goal of workers in this area. Achieving this goal requires compromises between the sophistication of solid-state physics and the pragmatic demands of electrical engineering. There are two classes of computer models:

(a) Compact models, which use the methods of Gummel-Poon or Ebers-Moll, based on closed form solutions to approximate device equations.

(b) Detailed models based on doping profiles and numerical solutions to the coupled nonlinear semiconductor device equations with appropriate boundary conditions [1]. These equations are usually solved self-consistently by either finite element or finite difference procedures and in-

\footnotetext{
The views stated in this paper are those of the author and do not represent necessarily the views of the National Bureau of Standards.
}

clude Poisson's equation, current-density equations for holes and electrons, and continuity equations for holes and electrons.

The discussion that follows pertains to detailed device models.

\section{Typical Device Structures}

Figure 1 gives the structure for a device similar to the one investigated by Asbeck et al. [2]. This HBT avoids many of the tradeoffs in the design of homojunction bipolar transistors. The HBT has a wider bandgap emitter (layer 3 ). The greater bandgap of the emitter compared to the base reduces substantially the hole injection from the base into the emitter. Reducing the basewidth decreases the electron transit time in the device and thereby increases $f_{\tau}$. The conduction band spike is reduced by the compositional grading [3]. This increases the injected electron current and, therefore, the gain of the HBT. Such HBT devices with thin bases and high $f_{\tau} \mathrm{s}$ are fabricated by molecular beam epitaxy (MBE) or by organometallic vapor phase epitaxy (OMVPE).

Circuits containing HBTs, which are similar to the HBT in Fig. 1, and operating at $300 \mathrm{~K}$ or $77 \mathrm{~K}$ may compete with or exceed the ultrahigh performance of Josephson junction circuits at 4K [4]. However, before HBTs can be used in high-speed integrated circuits, improved fabrication technology needs to be devised. In particular, the number of defects in the substrate and the number of interface states at junctions need to be reduced.

Figure 2 shows a typical heterostructure HFET, which is similar to the one reported in Ref. [5]. The main feature of these devices is to have the donors in a wider bandgap $\mathrm{GaAl}_{x} \mathrm{As}_{1-x}$ layer (layer 2) and to have the electrons move in a nearby undoped GaAs channel (layer 4). These devices frequently have a spacer layer of undoped GaAs (layer 3) to shield the two-dimensional electron gas from the fields of the donors in layer 2 . When the donor densities in layer 2 exceed about $10^{17} \mathrm{~cm}^{-3}$, the electrons do not have a bound state associated with the donor ions. The electron-ion scattering becomes significant in layer 2 and lowers the electron mobility. The undoped layers 3 and 4 provide the required high mobility, active region of the device.

The nonlinearity of the HFET is good for $L_{s} \mathrm{~s} \sim 1 \mu \mathrm{m}$ and very good for $L_{g} \mathrm{~s} \sim 0.5 \mu \mathrm{m}$ [4]. This nonlinearity is the increase of $g_{m}$ with small $\left(V_{g^{s}}-V_{T}\right)$ and increases as $\mu_{n} / L_{g}$ increases, where $\mu_{n}$ is the mobility of the electron in the channel. The current gain-band- 
width product increases as $L_{8}$ decreases. Hence, to increase $g_{m} s$ and $f_{\tau} s$, shorter gate lengths and higher mobilities are needed. Gate lengths less than $0.5 \mu \mathrm{m}$ place unacceptable demands on lithographic precision for commercial production. Heterostructure HFETs offer ways to achieve mobilities that are higher than those for GaAs alone. Such FETs are called TEGFETs [5], MODFETs, HEMTs [6], and SDFETs. One reason that similar devices are referred to by many names is that the understanding of how the devices function is incomplete. Most workers concentrate on how the carriers move in the channel and place less emphasis on how the carriers move from the source to the channel and from the channel to the drain.

\section{Representative Device Models and Associated Assumptions}

Many HBT, MESFET, and HFET models exist in the literature. Most authors use the predictions from such models to perform numerical experiments and thereby to suggest ways to improve device performance. Few compare the predictions with measurements from devices. In this section, five representative device models are presented to illustrate the recent advances in modeling GaAs devices. These five device models have been selected from among the many models reported in the archival literature, since as a set, they contain most of the essential features and assumptions found in HBT, MESFET, and HFET models. The many other models that are not discussed or referenced here are, with few exceptions, variations on those outlined below. Including discussions of additional models would detract from the main purposes of this review; namely, identifying areas for which improved physical concepts and measurement techniques are needed.

\section{Formulation of Lundstrom and Schuelke}

Lundstrom and Schuelke have developed a numerical method for analyzing heterostructure semiconductor devices (HBTs and HFETs)[7]. Their analysis is based on a macroscopic description of semiconductors with nonuniform composition. The LundstromSchuelke (LS) model contains conventional device equations. It discretizes the basic equations by the finite difference technique and uses the ScharfetterGummel [8] formulation for the current densities.

These authors have modified conventional device analysis programs to evaluate the two band parameters $V_{n}$ and $V_{p}$. They describe the nonuniform composition by position dependent $\kappa_{s}(\mathrm{x}), V_{n}(\mathrm{x})$, and $V_{p}(\mathrm{x})$. These modifications are valid only for heterostructures in equilibrium. Also, the LS model is strictly valid only when the material composition changes slowly and the concept of a position dependent effective mass is reasonable [9].

However, the above restrictions may not be appropriate for processed GaAs.
The main assumptions of the LS class of models are summarized below. The equilibrium $p n$ product is assumed to have the form

$$
n_{0} p_{0}=n_{i}^{2} \exp \left(-\Delta E_{g} / \kappa T\right)
$$

where $\Delta E_{g}=-q\left(V_{n}+V_{p}\right)$. Recent work [10] has shown that Eq. (1) gives incorrect descriptions for heavily doped silicon devices with emitter widths less than $3 \mu \mathrm{m}$. Preliminary results indicate that similar difficulties occur with Eq. (1) for GaAs above $10^{17} \mathrm{~cm}^{-3}$. Also, this and most other models assume that the temperature is uniform throughout the device and that no strains are present. However, Moglestue has shown that local heating occurs between the gate and drain of $n$-type GaAs FETs [11].

Lundstrom and Schuelke have applied their model to both the HBT in Ref. [2] and to the TEGFET in Refs. $[5,12]$. They have not compared the predictions of the LS model for HBTs with measured I-V data. Only numerical experiments to understand better the behavior of HBTs are reported. They have compared the LS model predictions with measured quasi-static capacitance versus reverse gate voltage for the TEGFET [12]. The agreement is good except at high voltages $(>8 \mathrm{~V})$, for which breakdown may occur.

\section{Formulation of Asbeck et al.}

Asbeck et al. [13] have modified the one-dimensional, finite difference code SEDAN [14] to be applicable for HBTs. The basic semiconductor equations are similar to those for the LS model in the "Formulation of Lundstrom and Schuelke," section except the constitutive relation for $\mathbf{J}_{n}$

$$
\mathbf{J}_{n}=-n q \mu_{n} \nabla\left(V+V_{n}\right)+D_{n} \nabla_{n}
$$

is replaced with the equation

$$
\mathbf{J}_{n}=-q n \mathbf{v}(\mathbf{x})
$$

The electron velocities are obtained from equilibrium and ballistic transport relations for $\mathbf{v}(\mathbf{E})$. They have considered the dependence of $f_{\tau}$ on current density and on various $\mathbf{v}(\mathrm{E})$ relations [15] by performing numerical experiments on HBTs [2]. They have used these predictions to suggest new designs for devices.

Because adequate measurements and theories for the dependence of $n_{i e}^{2} / n_{i}^{2}$ on the high carrier and dopant densities present in their HBTs do not exist, HBT models contain the assumptions that $n_{i e}^{2} / n_{1}^{2}=1$ and that $\mu_{p}(\mathrm{maj})=\mu_{p}(\mathrm{~min})$ and $\mu_{n}(\mathrm{maj})=\mu_{n}(\mathrm{~min})$ at the same doping densities. These physically questionable assumptions exist also in the recent Monte Carlo simulations reported by Tomizawa et al. [16].

Using the current crowding under the emitter as a variational parameter, Asbeck et al. have compared the dc common emitter gain versus collector current with measured values. The agreement is marginally acceptable. Continuing additional numerical experiments without better input data for mobilities, band 
edge changes, and effective intrinsic carrier concentrations has limited value to assist in improving HBTs.

\section{Formulation of Riemenschneider and Wang}

Riemenschneider and Wang have developed a twodimensional, finite-element program for analyzing transient and steady-state characteristics of GaAs MESFETs [17]. The devices that they have analyzed are dominated by electrons, and the contribution of holes to the total current flow is negligible. The holes would be important if the model were to include parasitic effects, such as backgating or other mechanisms for minority carrier injection. But, few, if any, twodimensional, finite-element models consider parasitics. The generation and recombination terms in the relation for the conservation of electrons are set to zero. The electron current in the model is derived from classical transport theory

$$
\mathbf{J}_{\mathbf{n}}=-q n \mathbf{v}_{\mathbf{n}}+D_{n} \nabla n
$$

Equation (4) contains transport by diffusion, whereas Eq. (3) does not.

The values of the electron velocity are based on steady-state data from the Monte Carlo calculations of Ref. [18]. The diffusivity $D_{n}$ values are interpolated between the low field values computed by Einstein's relationship and the high field values [19]. They do not include the effect of dynamic velocity overshoot. Since most velocity overshoot formulations such as Cook and Frey [20] do not consider the multivalley nature of electron transport in GaAs in detail, Riemenschneider and Wang consider the accuracy of the models that use them to be doubtful.

Riemenschneider and Wang have reported only numerical experiments on comparing the predicted performance of planar MESFETs with recessed gate MESFETs. They have no experimental verification of their calculations. This tends to be the rule and not the exception for modeling GaAs devices. Verification of models to the extent accomplished for silicon devices is rare for GaAs device models.

\section{Formulation of Cook and Frey}

Cook and Frey [20] have presented computer simulations of GaAs MESFETs that include transport effects (velocity overshoot). They offer an engineering-level description of hot electron effects in GaAs MESFETs. Their transport model contains many assumptions. Some of the more significant ones are as follows:

(1) Including the upper and lower valleys in the transport equations makes the procedure too complicated, so they have used an equivalent single valley model [21].

\section{List of Symbols}

D Defect or trap density

$D_{n} \quad$ Electron diffusivity

E Carrier energy

$E_{F} \quad$ Fermi energy

$E_{G} \quad$ Bandgap

E Electric field

$f_{r} \quad$ Current-gain bandwidth products

$\mathrm{g}_{m} \quad$ Transconductance

G Generation rate

$\hbar$ Planck's Constant $/ 2 \pi$

I-V Current-voltage characteristic

J Current density

k Boltzmann Constant

k Carrier wave vector

$L_{8} \quad$ Gate length

$m^{*} \quad$ Carrier effective mass

$\mathrm{n} \quad$ Electron density

$n_{0} \quad$ Electron equilibrium density

$n_{i} \quad$ Intrinsic carrier concentration

$n_{i e} \quad$ Effective intrinsic carrier concentration

$N_{D} \quad$ Donor density

$N_{A} \quad$ Acceptor density

$\mathrm{p}$ Hole density

$p_{0} \quad$ Hole equilibrium density

q Electronic charge
$\mathrm{R}$

t

$\mathrm{T}$

$V_{g s}$

$V_{B E}$

$V_{T}$

$V_{n}$ and $V_{p}$

W

$W_{B}$

$W_{E}$

$\mathbf{x}$

$\beta$

$\Gamma, \mathrm{L}, \mathrm{X}$

$\Delta_{\mathrm{rL}}$

$\Delta_{\Gamma X}$

$\epsilon$

$\kappa_{s}$

$\mu_{n}$ (maj)

$\mu_{n}(\min )$

$\mu_{p}$ (maj)

$\mu_{p}(\min )$

$\tau_{p}(\mathrm{~W})$

$\tau_{W}(W)$
Recombination rate

Time

Temperature

Gate-source voltage

Base-emitter voltage

Threshold voltage

Band parameters

Carrier energy

Base width

Emitter width

Position vector

Gain

Symmetry points in Brillouin Zone

Subband energy difference between $\Gamma$ and $L$ points

Subband energy difference between $\Gamma$ and $X$ points

Dielectric constant

Position-dependent dielectric constant

Electron mobility n-type

Electron mobility p-type

Hole mobility p-type

Hole mobility n-type

Momentum relaxation time

Energy relaxation time 
(2) The electron-phonon and electron-ionized impurity scattering processes can be described by energy-dependent relaxation times for electron momentum and energy, $\tau_{p}(W)$ and $\tau_{w}(W)$, respectively, i.e.,

$$
\begin{gathered}
(\mathrm{dv} / d t) \text { coll }=\mathrm{v} / \tau_{p}(\mathrm{~W}) \\
(d \mathrm{~W} / d t) \text { coll }=\left(\mathrm{W}-\mathrm{W}_{o}\right) / \tau_{w}(W)
\end{gathered}
$$

(3) Equations (5) and (6) require the assumption that $\tau_{p}\left(W\left(t_{1}\right)\right)=\tau_{p}\left(W\left(t_{2}\right)\right) \quad$ and $\quad \mathrm{wt}_{w}\left(W\left(t_{1}\right)\right)=$ $\tau_{w}\left(W\left(t_{2}\right)\right)$.

Most discussions of energy transport in GaAs use expressions such as those given by Eqs. (5) and (6). However, these expressions may not be valid for GaAs. Nougier et al. have shown in Ref. [22] that the transient velocity in a semiconductor is the solution of a relaxation time equation, such as Eq.(5), when impurity and polar (longitudinal long range) optical phonon scatterings are negligible. However, impurity and optical phonon scatterings [23] are large for the structures shown in Figs. 1 and 2.

Hence, a very significant question remains to be answered in the modeling of state-of-the-art GaAs devices. Namely, do the two assumptions (5) and (6) compensate each other and lead to good quantitative simulations or do they add and lead to additional errors? Before this question may be answered, however, the quantitative correctness of the boundary conditions used in GaAs device models must be determined. The convoluted nature of boundary conditions representing the physical device and of assumptions (5) and (6) makes verifying GaAs device models a challenge. Others [24] state that, at present, the boundary conditions used in most GaAs models may be quantitatively in much greater error than the errors associated with any of the above assumptions. If this is true, then comparing the predictions of energy transport and conventional models with measured I-V data is not of value until knowledge of the boundary conditions improves.

Cook and Frey have compared the predictions of conventional models with their energy transport model for the case of a uniformly doped planar MESFET. They have concluded that whenever $W(E)$ increases rapidly with $\mathrm{E}$, then the transport model should be quantitatively superior to conventional models. However, they have performed only numerical experiments.

\section{Formulation of Yoshii, Tomizawa, and Yokoyama}

Using a Monte Carlo scattering description, which is similar to that given by W. Hockney et al. [25], Yoshii, Tomizawa, and Yokoyama [26] follow in space and time the trajectories for all particles under the inhomogeneous, local electric field. Their method is a valid way to solve the Boltzmann transport equation. As is the case for the formulation of Cook and Frey, the formulation of Yoshii et al. requires that the scat- tering mechanisms and the band structure be quantitatively given as functions of carrier concentrations and/or doping densities. However, since it also requires substantial computer resources compared to the other methods, it is used rarely to optimize devices. Instead, full Monte Carlo particle simulations may provide a sound physical basis for parameters in the relaxation time approximation. The latter then may be used with improved confidence to optimize device performance.

Using their two-dimensional full Monte Carlo particle simulations, the authors of reference [26] have concluded that nonstationary carrier transport influences considerably the device characteristics of submicrometer-gate GaAs MESFETs and that the relaxation time approproximation may overestimate the nonstationary effects.

\section{Physical Concepts for GaAs Devices}

Reducing the number of unknown parameters in device models increases the effectiveness of computer models for product development. The extent to which manufacturers of GaAs ICs will be successful in "forward engineering" with computer models depends, in part, on the correctness of the physical concepts used. An example of "forward engineering" based on detailed models is the recently reported improvement in the performance of short-channel NMOS devices [27].

It is essential that any changes in the device physics be anticipated at the time that computer codes for solving, numerically, the device model are developed. The numerical stability of the solutions depends greatly on the algorithms employed [24]. The latter may be sensitive to the dependences of the device parameters on densities, electric fields, temperatures, and position.

The remaining parts of this section contain examples of how improved device physics might contribute to better performance of $\mathrm{GaAs} / \mathrm{AlGaAs} \mathrm{HBTs}$ and FETs.

\section{GaAs/AlGaAs Bipolar Heterostructures}

The potential for high-frequency performance has increased the effort devoted to GaAs/AlGaAs heterojunction bipolar transistors (HBT). Proposed devices [28] have $p^{+}$bases with Be acceptor densities of $10^{19} \mathrm{~cm}^{-3}$ and $n^{+}$emitters with $\mathrm{Si}$ donor densities of $5 \times 17^{17} \mathrm{~cm}^{-3}$. Estimates for $\mathrm{GaAs}$ indicate that its band structure is perturbed whenever dopant ion or carrier concentrations exceed $6 \times 10^{18} \mathrm{~cm}^{-3}$ in p-type GaAs and exceed $10^{17} \mathrm{~cm}^{-3}$ in n-type GaAs [29]. Above these concentrations, theory suggests that quantities such as $E_{G}, \Delta_{\tau L}, \Delta_{\tau X}, n_{i e}$ mobilities, and lifetimes should be calculated in terms of the perturbed band structures and not in terms of the band structure for the intrinsic material.

Any bipolar-detailed device model planned for the immediate application to GaAs/AlGaAs HBTs, such 


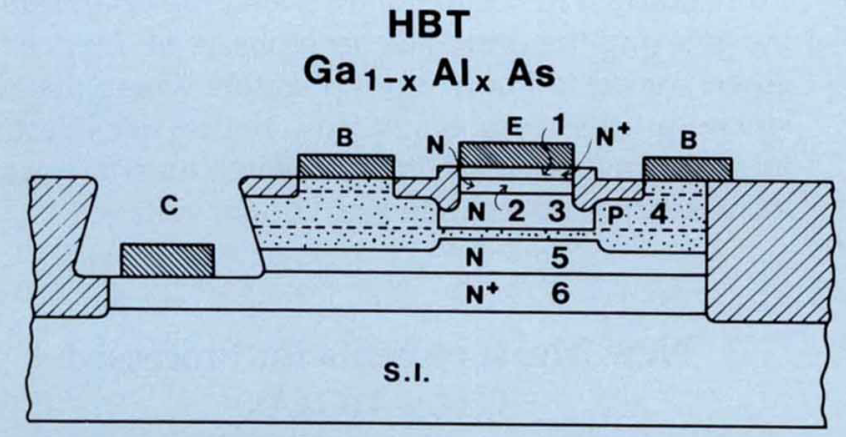

U//Ion bombardment damage isolation regions Be-implanted and base regions

Allmivionmic metallization

\begin{tabular}{|c|c|c|c|c|}
\hline Layer & $\begin{array}{l}N_{D}-N_{A} \\
\left(\mathrm{~cm}^{-3}\right) \\
\end{array}$ & $\begin{array}{c}\text { Al } \\
\text { Fraction } \\
\end{array}$ & $\begin{array}{l}\text { Thickness } \\
\quad(\mu \mathrm{m}) \\
\end{array}$ & Function \\
\hline 1 & $1 \times 10^{19}$ & 0 & 0.075 & cap \\
\hline 2 & $5 \times 10^{17}$ & 0 & 0.125 & cap \\
\hline 3 & $5 \times 10^{17}$ & $0-0.3$ & 0.03 & grading \\
\hline 3 & $5 \times 10^{17}$ & 0.3 & 0.22 & emitter \\
\hline 3 & $5 \times 10^{17}$ & $0.3-0$ & 0.03 & grading \\
\hline 4 & $-1 \times 10^{19}$ & 0 & 0.1 & base \\
\hline 5 & $1 \times 10^{16}$ & 0 & 0.5 & collector \\
\hline 6 & $2 \times 10^{18}$ & 0 & 0.2 & buffer \\
\hline
\end{tabular}

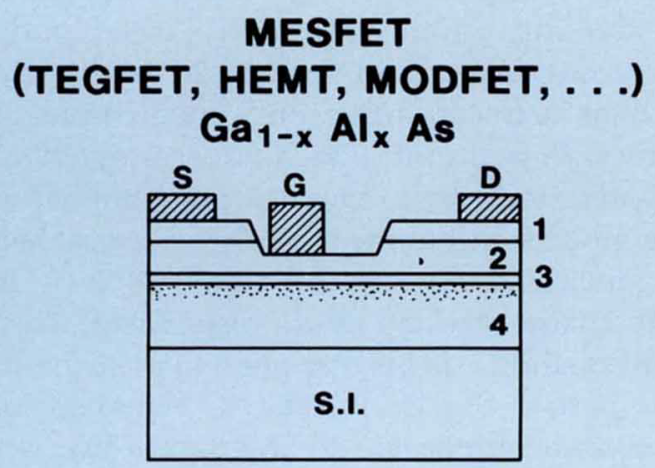

\begin{tabular}{|c|c|c|c|}
\hline Layer & $\begin{array}{l}N_{D}-N_{A} \\
\left(\mathrm{~cm}^{-3}\right) \\
\end{array}$ & $\begin{array}{c}\text { Al } \\
\text { Fraction } \\
\end{array}$ & $\begin{array}{l}\text { Thickness } \\
(\mu \mathrm{m})\end{array}$ \\
\hline 1 & $\lesssim 10^{18}$ & 0 & 0.02 \\
\hline 2 & $\sim 10^{17}-10^{18}$ & $\sim 0.3$ & 0.1 \\
\hline 3 & 0 & $0.3-0.7$ & 0.008 \\
\hline 4 & $\sim 0$ & $0.0-0.3$ & 1.0 \\
\hline & $\begin{array}{r}\text { S,D } \\
\text { G }\end{array}$ & $\begin{array}{l}\text { uGe-Ni } \\
\text { iAl }\end{array}$ & \\
\hline
\end{tabular}

Fig. 2 Cross section of the enhancement mode TEGFET described in Ref. 12. 
as those described in Ref. [13], would not incorporate these effects because of high dopant and carrier concentrations. This would occur because expressions for such quantities as $n_{i e}$ as a function of doping or carrier density are not available in a form suitable for use in detailed models. The usual expressions for the majority carrier mobilities and drift velocities would also be used, because acceptable expressions for minority carriers do not exist. A third area of difficulty is that knowledge of recombination centers and minority carrier lifetimes is very meager in GaAs. For example, if the number of recombination centers is too great, carrier transport becomes impeded. As a result of this lack of verified input data for $\mathrm{GaAs}$ bipolar device models, no discernible correlation between measurements and model predictions should be expected.

\section{FETS}

Numerical simulations for the carrier transport in the channel of MESFETs reveal three distinct effective field regions: contact, channel, and a rapidly forming space charge dipole [30]. For a $1 \mu \mathrm{m}$-gate with an n-type dopant density of $2 \times 10^{17} \mathrm{~cm}^{-3}$ in the channel of a conventional MESFET, the carrier density in the accumulation region of the dipole region is about $3.5 \times 10^{17} \mathrm{~cm}^{-3}$ and the carrier density in the depletion region is about $10^{17} \mathrm{~cm}^{-3}$. This dipole forms in about 10ps when ideal voltage sources are assumed [31].

Device performance characteristics, such as transconductance, are sensitive to changes in the carrier mobility. The changes in mobilities for the channel and dipole regions depend in part on the band structure parameters, particularly on $\Delta_{\Gamma L}[29,24]$. These band parameters are expected to differ from the intrinsic band parameters due to carrier-dopant ion and carrier-carrier interactions.

Backgating and light sensitivity, which degrade circuit performance and may limit packing densities $[32,33]$ in MESFETs, have been shown by C. P. Lee and coworkers $[34,35]$ to be related to carrier injection from the substrate and to carrier transport from the substrate to the active region. Many proposals for reducing the effects of backgating $[34,36,37]$ involve the transport of minority carriers.

The source and drain contacts and the Schottkybarrier gate provide other areas where physical concepts are not adequate. For example, the transition from the n-type channel, through the $n^{+}$drain, to the highly degenerate alloy contact has abrupt concentration changes and large mobility variations. Also, the presence of trapping states at the Schottky-barrier interface greatly influences the Fermi energy and the work function and may contribute a new mechanism of charge storage. The latter may limit device speed.
Design engineers need detailed models for ohmic contacts and Schottky barriers to predict their highfrequency behavior. They are particularly interested in knowing before fabrication whether any upper limits for the frequency responses of contacts or gates will affect their design considerations.

Breakdown in the region between the drain side of the gate and the drain has been observed. Device engineers need to know more precisely where the avalanche process begins and how the carrier injection mechanism takes place in their search for strategies to increase the gate to drain breakdown voltage.

\section{New Measurements for Processed GaAs/AlGaAs}

As devices become faster and smaller, understanding the ultrafast behavior and nonequilibrium transport must improve. Designers of high-speed devices lack accurate techniques to measure device performance. An underlying principle is that the measurement technique must be faster than the device under test. Electrical measurements are usually used to test devices. This approach is successful when the electrical techniques for device characterization are faster than the devices under development. However, when the goal is to build the fastest device, the usual electrical techniques use the device to measure itself. An example of the latter approach is the ring oscillator technique applied to MESFETs. Since the time resolution of the electrical measurements based on a ring oscillator is determined by the individual devices themselves, only estimates of device speed are possible and little understanding of ultrafast processes and nonequilibrium transport result.

Pulsed optical or electron beam techniques offer alternative methods by which to measure many of the parameters listed in the Appendix and to increase understanding ultrafast devices. Pulsed optical techniques are faster than most high-speed technologies such as semiconductor and superconducting electronics. Pulsed optical techniques may, therefore, be the preferred way to measure key parameters for device models and to measure the electrical behavior of fast devices. One new technique [38] introduces discrete charge packets by a pulsed laser. This time of flight method has been applied to measure high-field transport at $\mathrm{Si}_{-} \mathrm{SiO}_{2}$ interfaces. Whether such techniques are applicable to interfaces involving $\mathrm{GaAs}$ needs to be determined. A basic problem is that a technology for building a resistive gate in GaAs does not exist. Other challenges include: (1) pulsed optical or electron beam techniques may not be fast enough for GaAs; (2) sample quality may not be adequate over the path length needed [24]; and (3) applying a uniform, lateral electric field to the sample over the entire path length has not been demonstrated for GaAs. 


\section{Conclusions}

The major conclusions from the discussions above are

(1) The measured and theoretical data for many of the electrical and material properties of GaAs devices are not adequate for reliable engineering without detailed verification by measurements. The input quantities for detailed device models contain many unknown parameters.

(2) The interdependence of numerical stability and device physics dictates that any changes in the device physics must be anticipated at the time the algorithms and computer codes are developed.

(3) Many physical concepts are not adequate for GaAs devices. These include carrier scattering rates due to ionized impurities and polar optical phonons and carrier transport when carrier or doping concentrations exceed $10^{17} \mathrm{~cm}^{-3}$ in n-type and $10^{19} \mathrm{~cm}^{-3}$ in p-type $\mathrm{GaAs} / \mathrm{AlGaAs}$. Incorporating adequate physical concepts in GaAs device models requires new measurement techniques, perhaps based on ultrafast spectroscopy, for mobilities and lifetimes as functions of electric fields, dopant density, carrier energy, and carrier density in processed GaAs. Specially designed test structures will be needed to resolve many of the uncertainties concerning the physical concepts to be incorporated into GaAs device models.

\section{Appendix: Input Parameters for GaAs Device Modeling}

Several of the input parameters that are needed for modeling GaAs devices are listed below.

\section{Band Structure}

Bandgap $E_{G}\left(N_{D}\right)$ and valley separations $\Delta_{\Gamma L}\left(N_{D}\right)$ and $\Delta_{\Gamma x}\left(N_{D}\right)$

Equilibrium Carrier Densities

$n_{i e}^{2}\left(N_{D}\right)=n p$ and $n_{i e}^{2}\left(N_{A}\right)=n p$

Transport Parameters for Majority and Minority Carriers $\mu_{n}\left(\mathbf{E}, N_{D}\right)$ and $\mu_{n}\left(\mathbf{E}, N_{A}\right)$

$v_{n}\left(\mathbf{E}, N_{D}\right)$ and $v_{n}\left(\mathbf{E}, N_{A}\right)$

$\mu_{p}\left(\mathbf{E}, N_{D}\right)$ and $\mu_{p}\left(\mathbf{E}, N_{A}\right)$

$v_{p}\left(\mathbf{E}, N_{D}\right)$ and $v_{p}\left(\mathbf{E}, N_{A}\right)$

where $E$ is the electric field

\section{Recombination Parameters}

Auger recombination lifetimes for holes and electrons

Shockley-Read-Hall (defect) lifetimes for holes and electrons

\section{Generation Parameters}

Impact ionization rates and tunnel rates

$$
\text { Dopant Density Profiles }
$$

$N_{D}(x, y, z)$ and $N_{A}(x, y, z)$

\section{Defect Density Profiles}

$D(x, y, z)$ and the extent to which $D(x, y, z)$ depends on $N_{D}$ and $N_{A}$

Contact and Interface Parameters

Interface trap density, Fermi energy, and work function at Schottky-barrier interface

Interface trap densities at ohmic contacts and other interfaces

\section{Acknowledgments}

The author thanks the staff members of the Semiconductor Devices and Circuits Division who have provided helpful comments during the preparation of this article.

\section{References}

[1] J. L. Blue and C.L. Wilson, "Two-Dimensional Analysis of Semiconductor Devices Using General Purpose PDE Software," IEEE Trans. Electron Devices, vol. 30, p. 1056, 1983.

[2] P. Asbeck, D. L. Miller, R. A. Milano, J. S. Harris, G. R. Kaelin, and R. Zucca, "(Ga,A1)As/GaAs Bipolar Transistors for Digital Integrated Circuits," Technical Digest, 1981 International Electronic Devices Meeting (IEEE, New York), pp. 629-632.

[3] H. Kroemer, "Heterostructure Bipolar Transistor and Integrated Circuits," Proc. IEEE, vol. 70, p. 13, 1982.

[4] R. C. Eden, "Comparison of GaAs Device Approaches for Ultrahigh-Speed VLSI," Proc. IEEE, vol. 70, p. 5, 1982.

[5] D. Delagebeaudeuf, P. Delescluse, P. Etienne, M. Laviron, J. Chaplant, and N. T. Linh, "Two-Dimensional Electron Gas MESFET Structure," Electron. Lett., vol. 16, p. 667, 1980.

[6] T. Mimura, S. Hiyamizu, and K. Nanbu, "A New Field-Effect Transistor with Selectively Doped GaAs/n- $\mathrm{Al}_{\mathrm{x}} \mathrm{Ga}_{1-\mathrm{x}} \mathrm{As}$ Heterojunctions," Jap. J. Appl. Phys. Lett., vol. 19, p. 225, 1980.

[7] M. S. Lundstrom and R. J. Schuelke, "Numerical Analysis of Heterostructure Semiconductor Devices," IEEE Trans. Electron Devices, ED-30, p. 1151, 1983.

[8] D. L. Scharfetter and H. K. Gummel, "Large-Signal Analysis of a Silicon Read Diode Oscillator," IEEE Trans. Electron Devices, ED-16, p. 64, 1969.

[9] K. M. van Vliet and A. H. Marshak, "Wannier-Slater Theorem for Solids With Position Dependent Band Structure," Phys. Rev., B26, p. 6734, 1982.

[10] H. S. Bennett, "Improved Concepts for Predicting the Electrical Behavior of Bipolar Structures in Silicon," IEEE Trans. Electron Devices, ED-30, p. 920, 1983.

[11] C. Moglestue, IEE Proc., vol. 128, p. 131, 1981.

[12] M. Le Brun, P. R. Jay, C. Rumelhard, G. Rey and P. Delescluse, "Low Noise Performance of Two-Dimensional Electron Gas FETs," Proc. IEEE/Cornell Conference on High-Speed Semiconductor Devices and Circuits, IEEE Cat. No. 83CH1959-6, p. 187, Aug. 1983.

[13] P. M. Asbeck, D. L. Miller, R. Asatourian, and C. G. Kirkpatrick, "Numerical Simulation of GaAs/AlGaAs Heterojunction Bipolar Transistors," IEEE Electron Device Lett., EDL-3, p., 402, 1982.

[14] Semiconductor Device Analysis (Stanford University), Jan. 1980 version. Certain materials and computer codes are identified in this paper in order to specify the procedures adequately. Such identification does not imply recommendation or endorsement by the National Bureau of Standards, nor does it imply that the materials or computer codes identified are necessarily the best available for the purpose.

[15] S. Katzer and J. Frey, "Transient Velocity Characteristics of Electrons in GaAs With $\Gamma$-L-X Conduction Band Ordering," J. Appl. Phys., vol. 49, p. 4064, 1978. 
[16] K. Tomizawa, Y. Awano, and N. Hashizume, "Monte Carlo Simulation of $\mathrm{AlGaAs} / \mathrm{GaAs}$ Heterojunction Bipolar Transistors," IEEEE Electron Device Lett., EDL-5, p. 362, 1984.

[17] P. R. H. Riemenschneider and K. L. Wang, "A Finite-Element Program for Modeling Transient Phenomena in GaAs MESFETs," IEEE Trans. Electron Devices, ED-30, p. 1142, 1983.

[18] M. A. Littlejohn, J. R. Hauser, and T. H. Glisson, "VelocityField Characteristics of GaAs with $\Gamma$-L-X Conduction Band Ordering," J. App. Phys., vol. 48, p. 4587, 1977.

[19] J. Pozela and A. Reklaitis, "Electron Transport Properties in GaAs at High Electric Fields," Solid-State Electronics, vol. 23, p. $927,1980$.

[20] R. K. Cook and J. Frey, "Two-Dimensional Numerical Simulation of Energy Transport Effects in Si and GaAs MESFETs," IEEE Trans. Electron Devices, ED-29, p. 970, 1982.

[21] D. E. McCumber and A. G. Chynoweth, "Theory of Negative Conductance Amplication and of Gunn Instabilities in "Two Valley' Semiconductors," IEEE Trans. Electron Devices, ED-13, p. 4, 1966.

[22] J. P. Nougier, J. C. Vaissiere, and D. Gasquet, "Determination of Transient Regime of Hot Carriers in Semiconductors, Using the Relaxation Time Approximations," J. Appl. Phys., vol. 52, p. 825, 1981.

[23] J. W. Harrison and J. R. Hauser, "Theoretical Calculations of Electron Mobility in Ternary III-V Compounds," J. Appl. Phys., vol. 47, p. 292, 1976.

[24] C. L. Wilson, "High Accuracy Physical Modeling of Submicron MOSFET's," IEEE Trans. Electron Devices, ED-30, p. 1579, 1983.

[25] R. W. Hockney, R. A. Warriner, and M. Reiser, "Two-Dimensional Particle Models in Semiconductor-Device Analysis," Electron. Lett., vol. 10, p. 484, 1974.

[26] A. Yoshii, M. Tomizawa, and K. Yokoyama, "Accurate Modeling for Submicrometer-Gate Si and GaAs MESFET's Using Two-Dimensional Particle Simulation," IEEE Trans. Electron Devices, ED-30, p. 1376, 1983.

[27] W. Fichtner, "Physics and Simulation of Small MOS Devices," Technical Digest, 1982 International Electron Devices Meeting (IEEE, New York), p. 638.

[28] P. M. Asbeck, D. L. Miller, W. C. Petersen and C. G. Kirkpatrick, "GaAs/AIGaAs Heterojunction Bipolar Transistors with Cutoff Frequencies Above $10 \mathrm{GHz}$," IEEE Electron Device Lett., EDL-3, p. 366, 1982.

[29] H. S. Bennett, to be published.

[30] S. Swierkowski and L. F. Jelsma, "Two-Dimensional Computer Modeling of GaAs Devices," IEEE Trans. Electron Devices, ED-28, p. 1219, 1981.

[31] S. Swierkowski, private communication.

[32] M. S. Birrittella, W. C. Seelbach, and H. Goronkin, "The Effect of Backgating on the Design and Performance of GaAs Digital Integrated Circuits," IEEE Trans. Electron Devices, ED-29, p. 1135, 1982.

[33] H. Goronkin, M. S. Birrittella, W. C. Seelbach, and R. Vaitkus, "Backgating and Light Sensitivity in Ion-Implanted GaAs Integrated Circuits," IEEE Trans. Electron Devices, ED-29, p. 845, 1982.

[34] C. P. Lee and B. M. Welch, "GaAs MESFETs with Partial p-Type Drain Regions," IEEE Trans. Electron Devices, ED-29, p. 1687, 1982 and "GaAs MESFETs with Partial p-Type Drain Regions," IEEE Electron Device Lett., EDL-3, p. 200, 1982.

[35] C. P. Lee, S. J. Lee, and B. M. Welch, "Carrier Injection and Backgating Effects in GaAs MESFETs," IEEE Electron Device Lett., EDL-3, p. 97, 1982.

[36] C. Kocot and C. A. Stolte, "Backgating in GaAs MESFETs," IEEE Trans. Electron Devices, ED-29, p. 1059, 1982.

[37] D. C. D'Avanzo, "Proton Isolation for GaAs Integrated Circuits," IEEE Trans. Elecron Devices, ED-29, p. 1051, 1982.

[38] J. A. Cooper, Jr. and D. F. Nelson, "High-Field Drift Velocity of Electrons at the $\mathrm{Si}_{-} \mathrm{SiO}_{2}$ Interface as Determined by a Time-ofFlight Technique," J. Appl. Phys., vol. 54, p. 1445, 1983.

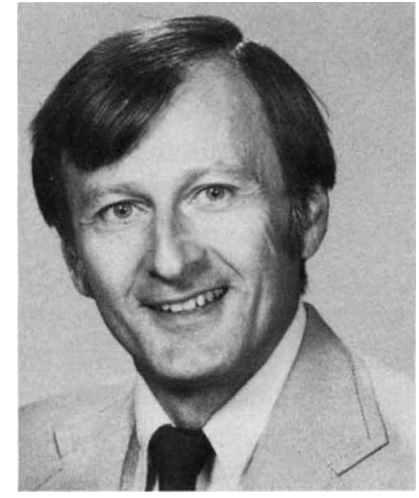

Herbert S. Bennett

From 1964 to 1966, Herbert S. Bennett (M '72) served as a Research Associate with the Atomic Energy Research Establishment (England) and with the University of Illinois, Department of Physics and Materials Research Laboratory. In September 1966, he accepted a position as Physicist with the National Bureau of Standards (NBS). He served in this capacity until September 1971, at which time he began his Science and Technology Fellowship assignment as a Special Assistant to the Assistant Secretary for Science and Technology in the Department of Commerce. He returned to NBS in July 1972 as Chief of the Solid-State Materials Section. From May 1978 to October 1979, he served as Director of the Division of Materials Research at the National Science Foundation. Dr. Bennett is currently a Senior Scientist and Group Leader for the Device and Process Modeling Group in the Semiconductor Devices and Circuits Division at NBS, Gaithersburg, MD 20899. His research interests have been primarily theoretical problems in electrical engineering and in the materials sciences.

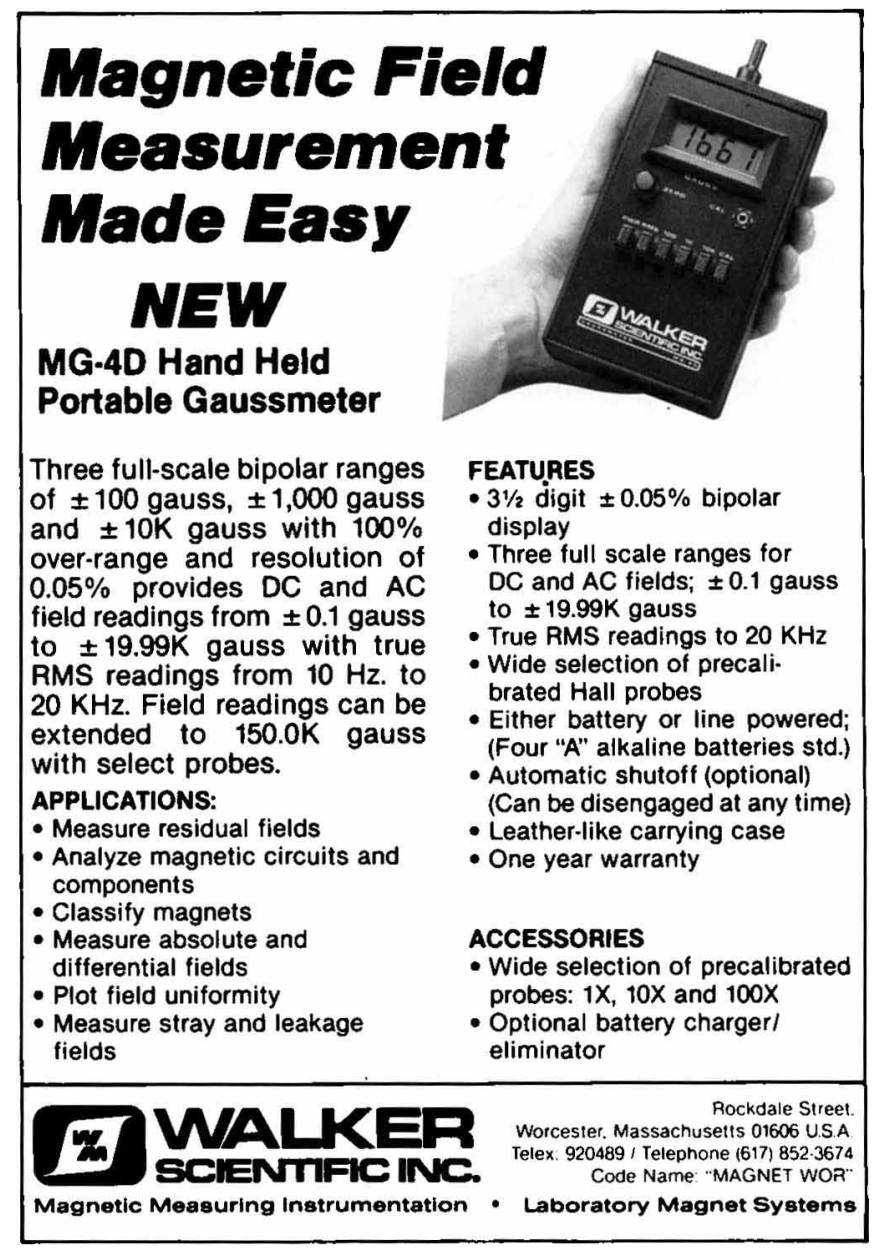


IEEE

Announces the 13th Annual Competition for

\section{대요 * \\ 1985-1986}

\section{Congressional Fellowships}

\section{A CONGRESSIONAL INTERNSHIP FOR MEMBERS OF IEEE}

PROGRAM: Electrical and Electronics Engineers and Allied Scientists are competitively selected to serve a one-year term on the personal staff of individual Senators or Representatives or on the professional staff of Congressional Committees. The program includes an orientation session with other Science-Engineering Fellows sponsored by the American Association for the Advancement of Science (AAAS).

PURPOSE: To make practical contributions to more effective use of scientific and technical knowledge in government, to educate the scientific communities regarding the public policy process, and to broaden the perspective of both the scientific and governmental communities regarding the value of such science-government interaction.

CRITERIA: Fellows shall be selected based on technical competence, on ability to serve in a public environment and on evidence of service to the Institute and the profession. Specifically excluded as selection criteria shall be age, sex, creed, race, ethnic background, and partisan political affiliations. However, the Fellow must be a U.S. citizen at the time of selection and must have been in the IEEE at Member grade or higher for at least four years. Additionaleriteria may be established by the selection committee.

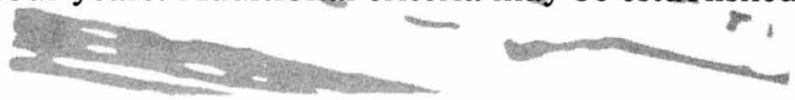

AWARDS: IEEE plans to award two Congressional Fellowships for the 1985-1986 term. Additional funding sources may permit expansion of awards.

APPLICATION: Further information and application forms can be obtained by calling W. Thomas Suttle (202) 785-0017 at the IEEE Washington, D.C. Office or by writing:

Secretary, Congressional Fellows Program

The Institute of Electrical and Electronics Engineers, Inc.

1111 Nineteenth St., N.W. Suite 608

Washington, D.C. 20036

Applications must be postmarked no later than March 30, 1985 to be eligible for consideration. 


\section{JOINT SPECIAL ISSUE \\ of the IEEE Transactions on Circuits and Systems and the IEEE Journal of Solid-State Circuits on \\ VLSI Analog and Digital Signal Processing-February 1986}

The purpose of this special issue is to focus on the algorithms, structures, architectures, and design methodologies that lead to economical VLSI implementations of analog and digital signal processing devices. A particular objective is to consider the cross elasticities, advantages, disadvantages, and competition between analog and digital implementations of VLSI signal processing functions for a variety of applications. An equally important objective is to explore the effects of VLSI design rule scaling on the evolution of these issues.

The Guest Editors for this special issue are:

Professor Kenneth R. Laker, Chair

Department of Electrical Engineering

School of Engineering and Applied Science

University of Pennsylvania

Philadelphia, PA 19104

(215) 898-8117

Professor Adel S. Sedra

Department of Electrical Engineering

University of Toronto

Toronto, Ontario

Canada, M5S $1 \mathrm{~A} 4$

(416) $978-6344$
Original research papers, state-of-the-art survey papers, and tutorial papers are solicited in the following areas:

- VLSI analog and digital filters

- A/D and D/A converters

- Custom and programmable DSPs

- VLSI design methodology

- Design rule scaling of analog and digital circuits

- VLSI applications to include but not restricted to:

1. echo cancellation/adaptive filters

2. speech synthesis/recognition

3. encription/desciption

4. video and image processing

5. high definition TV

6. high fidelity audio

7. telecommunications

Manuscripts are to be submitted for review to Professor Kenneth Laker before April 1, 1985. All manuscripts should conform to standard format as indicated in the "Information for Authors" printed on the back cover of the IEEE Circuits and Systems Transactions (the inside cover of the IEEE Solid-State Circuits Journal).

\section{WILL YOUR HI-REL PART PASS
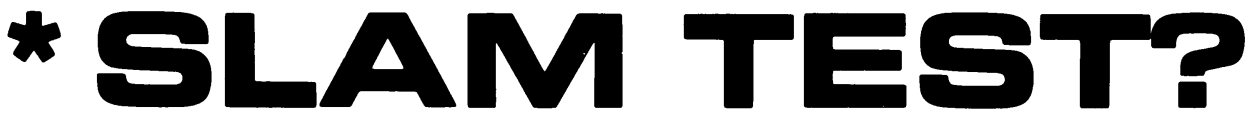

\section{* Scanning Laser Acoustic Microscope}

RAPID, NONDESTRUCTIVE INTERNAL INSPECTION OF FINISHED COMPONENTS

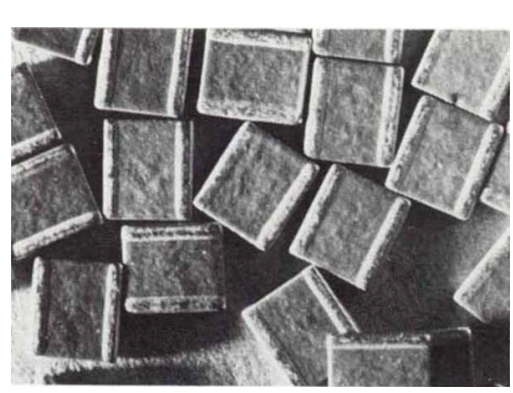

CERAMIC CAPACITORS

$$
\begin{gathered}
\text { [ALL TYPES] } \\
\text { Detection of- } \\
\text { cracks } \\
\text { delaminations } \\
\text { voids, inclusions } \\
\text { porosity } \\
\text { internal defects }
\end{gathered}
$$

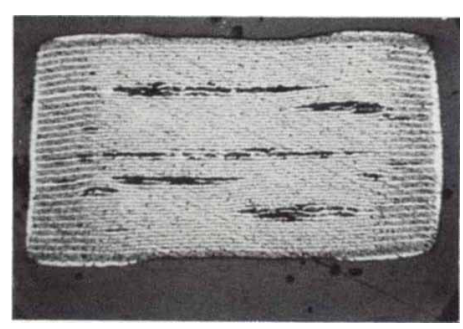

This capacitor passed extensive electrical testing. It failed the SLAM test.

Send us a sample. . . or 5,000 of them. We'll return the good ones, and the bad ones. We'll show you the differences - acoustically, and most importantly, NONDESTRUCTIVELY. For more information regarding SLAM testing for MIL-C-123 requirements, please contact our marketing department:

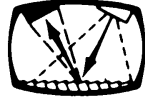

